\title{
Shrinkage Testimation in Exponential Distribution based on Records under Asymmetric Squared Log Error Loss
}

\author{
M. Naghizadeh Qomi* and L. Barmoodeh \\ University of Mazandarn
}

Received: 1/9/2016 Approved: 6/1/2016

\begin{abstract}
In the present paper, we study shrinkage testimation for the unknown scale parameter $\theta>0$ of the exponential distribution based on record data under the asymmetric squared log error loss function. A minimum risk unbiased estimator within the class of the estimators of the form $c T_{m}$ is derived, where $T_{m}$ is the maximum likelihood estimate of $\theta$. Some shrinkage testimators are proposed and their risks are computed. The relative efficiencies of the shrinkage testimators with respect to a minimum risk unbiased estimator of the form $c T_{m}$ under the squared log error loss function are calculated for the comparison purposes. An illustrative example is also presented.
\end{abstract}

Keywords. Digamma function; exponential distribution; records; shrinkage testimators.

MSC 2010: 62F03; 62F10.

${ }^{*}$ Corresponding author 


\section{Introduction}

Let $\left\{X_{i}, i \geqslant 1\right\}$ be a sequence of independent and identically distributed (i.i.d.) random variables having a cumulative distribution function (c.d.f.) $F$ and a probability density function (p.d.f.) $f$. An observation $X_{j}$ is said to be an upper record value if its value exceeds that of all previous observations. Thus, $X_{j}$ is an upper record if $X_{j}>X_{i}$ for every $i<j$. By convention $X_{1}$ is a record value. An analogous definition deals with lower record values. Data of this type arise in a wide variety of practical situations. Examples of application areas include industrial stress testing, meteorological analysis, sporting and athletic events, and oil and mining surveys; see Arnold et al. (1998) for these types of applications. We denote the $m$ th upper record value by $R_{m}$. The joint density of the first $m$-records $\mathbf{R}=\left(R_{1}, \ldots, R_{m}\right)$ is given by

$$
f_{R_{1}, \ldots, R_{m}}\left(r_{1}, \ldots, r_{m}\right)=f\left(r_{m}\right) \prod_{i=1}^{m-1} \frac{f\left(r_{i}\right)}{1-F\left(r_{i}\right)}, \quad r_{1}<r_{2}<\cdots<r_{m} .
$$

Also, the marginal p.d.f. of the $m$ th record, $R_{m}$, is given by

$$
f_{R_{m}}(x)=\frac{[-\log (1-F(x))]^{m-1}}{(m-1) !} f(x) .
$$

Throughout the paper, we denote by $\operatorname{Exp}(\theta)$ an exponential distribution with p.d.f.

$$
f(x ; \theta)=\frac{1}{\theta} \exp \left(-\left\{\frac{x}{\theta}\right\}\right), \quad x>0, \quad \theta>0 .
$$

If $\mathbf{R}=\left(R_{1}, \ldots, R_{m}\right)$ be the first $m$-records samples from the $\operatorname{Exp}(\theta)$-distribution, then from (1) and (3), the likelihood function of $\theta$ based on $\mathbf{R}=\left(R_{1}, \ldots, R_{m}\right)$ at $\mathbf{r}=\left(r_{1}, \ldots, r_{m}\right)$ is given by

$$
L(\theta \mid \mathbf{r})=\frac{1}{\theta^{m}} \exp \left(-\left\{\frac{r_{m}}{\theta}\right\}\right), \quad \theta>0 .
$$

Then, the MLE of $\theta$, denoted by $T_{m}$, can be derived from the equation $\frac{\partial L(\theta \mid \mathbf{r})}{\partial \theta}=0$, which is given by $T_{m}=R_{m} / m$. Also, by substituting the p.d.f. and the c.d.f. of the $\operatorname{Exp}(\theta)$-distribution in (2), the marginal p.d.f. of the $m$ th record, $R_{m}$, is given by 


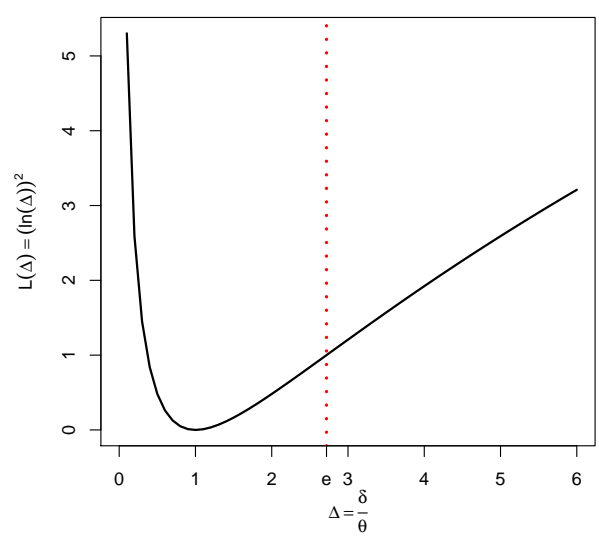

Figure 1. Plot of the SLEL function

$$
f_{R_{m}}(x)=\frac{x^{m-1} \exp \left(-\left\{\frac{x}{\theta}\right\}\right)}{\Gamma(m) \theta^{m}}, \quad \theta>0
$$

which implies that $R_{m} \sim \operatorname{Gamma}(m, \theta)$ and then $2 m T_{m} / \theta=2 R_{m} / \theta \sim \chi_{2 m}^{2}$.

Brown (1990) proposed Squared Log Error Loss (SLEL) function for estimating the scale parameter $\theta$ as

$$
L(\theta, \delta)=(\ln \delta-\ln \theta)^{2}=\left[\ln \frac{\delta}{\theta}\right]^{2},
$$

where both $\theta$ and $\delta$ are positive; see also Pal and Ling (1996). This loss is not symmetric and convex; it is convex for $\Delta=\frac{\delta}{\theta} \leqslant e$ (Euler's number) and concave otherwise, but has a unique minimum at $\Delta=1$. Also when $\Delta>1$, this loss increases sublinearly, while when $0<\Delta<1$, it rises rapidly to infinity at zero; see Figure 1. The SLEL function is useful in situations where underestimation is more serious than overestimation; see Sanjari Farsipour and Zakerzadeh (2005) and Kiapour and Nematollahi (2011).

According to Thompson (1968), a shrinkage estimator for the parameter $\theta$ when a prior point guess value $\theta_{0}$ of $\theta$ is available, is given by

$$
\hat{\theta}_{S}=k T_{m}+(1-k) \theta_{0}, \quad 0 \leqslant k \leqslant 1,
$$


where $k$ is a shrinkage factor. The value of $k$ near to zero (one) implies strong belief in the guess value $\theta_{0}$ (sample values). It seems that for the values of $\theta$ near to $\theta_{0}$, the shrinkage estimators should have performance better than the usual estimator $T_{m}$. Then, a preliminary test $H_{0}: \theta=\theta_{0}$ versus $H_{1}: \theta \neq \theta_{0}$ is performed for that $\theta_{0}$ is near to $\theta$ or not. For testing the hypothesis $H_{0}: \theta=\theta_{0}$ against $H_{1}: \theta \neq \theta_{0}$, a likelihood ratio test (LRT) statistic is $U=2 m T_{m} / \theta \sim \chi_{2 m}^{2}$ that has a rejection region of the form $U>q_{2}$ or $U<q_{1}$, where $q_{1}=\chi_{\alpha / 2,2 m}^{2}$ and $q_{2}=\chi_{1-\alpha / 2,2 m}^{2}$ are left quantiles of the chi-square distribution with $2 m$ degrees of freedom.

To this end, we propose some shrinkage testimators for the scale parameter of the $\operatorname{Exp}(\theta)$-distribution based on record data and study the performance of these testimators with respect to a minimum risk unbiased estimator within the class $c T_{m}$ under the SLEL function. A real data set is used for illustrating the results. Finally, we end the paper with some remarks.

\section{A Minimum Risk Unbiased Estimator of the Form $c T_{m}$}

Consider a class of estimators for the MLE of $\theta, T_{m}$, of the form $c T_{m}$. If $U=2 m T_{m} / \theta \sim \chi_{2 m}^{2}$, then the risk of $c T_{m}$ under the SLEL function is

$$
\begin{aligned}
R\left(\theta, c T_{m}\right) & =E\left[\ln \left(\frac{c T_{m}}{\theta}\right)\right]^{2}=E\left[\ln \left(\frac{c}{2 m} U\right)\right]^{2} \\
& =\ln ^{2}\left(\frac{c}{2 m}\right)+E\left[\ln ^{2}(U)\right]+2 \ln \left(\frac{c}{2 m}\right) E[\ln (U)] .
\end{aligned}
$$

Following Sanjari Farsipour and Zakerzadeh (2005), we have

$$
E[\ln (U)]=\ln (2)+\Psi(m), \quad E\left[\ln ^{2}(U)\right]=[\ln (2)+\Psi(m)]^{2}+\Psi^{\prime}(m),
$$

where $\Psi(m)=\frac{d}{d m} \ln \Gamma(m)=\frac{\Gamma^{\prime}(m)}{\Gamma(m)}$ is the digamma function, $\Psi^{\prime}(m)=$ $\frac{d}{d m} \Psi(m)$ is the trigamma function and $\Gamma(m)$ denotes the complete gamma function given by

$$
\Gamma(m)=\int_{0}^{\infty} t^{m-1} e^{-t} d t
$$


Upon substituting Equation (7) into Equation (6), we have

$$
\begin{aligned}
R\left(\theta, c T_{m}\right) & =\ln ^{2}\left(\frac{c}{2 m}\right)+[\ln (2)+\Psi(m)]^{2}+\Psi^{\prime}(m) \\
& +2 \ln \left(\frac{c}{2 m}\right)[\ln (2)+\Psi(m)] .
\end{aligned}
$$

The risk function in (8) is a convex function of $c$ and is minimized at the point $c=c_{1}$ given by

$$
c_{1}=m e^{-\Psi(m)} .
$$

Therefore, $c_{1} T_{m}$ is a minimum risk estimator of $\theta$ under the class $c T_{m}$ with finite risk as follows

$$
R\left(\theta, c_{1} T_{m}\right)=\Psi^{\prime}(m) .
$$

Following the definition of Lehmann (1951) an estimator $\delta$ of $\theta$ is said to be risk unbiased if it satisfies

$$
E[L(\theta, \delta)] \leqslant E\left[L\left(\theta^{\prime}, \delta\right)\right], \quad \forall \theta^{\prime} \neq \theta .
$$

Under the SLEL, we have

$$
E\left[\ln ^{2} \frac{\delta}{\theta}\right]-E\left[\ln ^{2} \frac{\delta}{\theta^{\prime}}\right]=\left(\ln ^{2} \theta-\ln ^{2} \theta^{\prime}\right)-2\left(\ln \theta-\ln \theta^{\prime}\right) E[\ln \delta] .
$$

If we consider $E[\ln \delta]=\ln \theta$, we conclude that

$$
E\left[\ln ^{2} \frac{\delta}{\theta}\right]-E\left[\ln ^{2} \frac{\delta}{\theta^{\prime}}\right]=-\left(\ln \theta-\ln \theta^{\prime}\right)^{2}<0 .
$$

Therefore, an estimator $\delta$ of $\theta$ is risk unbiased under the SLEL if it satisfies in the condition $E[\ln \delta]=\ln \theta$. Now, the estimator $c_{1} T_{m}$ satisfies the risk unbiased condition, as follows:

$$
\begin{aligned}
E\left[\ln \left(c_{1} T_{m}\right)\right] & =\ln \left(c_{1}\right)+E\left[\ln \left(T_{m}\right)\right]=\ln m-\Psi(m)+E\left[\ln \left(\frac{\theta U}{2 m}\right)\right] \\
& =\ln m-\Psi(m)+E[\ln (U)]-\ln 2-\ln m+\ln \theta \\
& =\ln \theta \quad(\text { using (7)) }
\end{aligned}
$$


Then, the estimator $c_{1} T_{m}$ is a minimum risk unbiased estimator under the class of $c T_{m}$.

Note that using Theorem 3.1 of Sanjari Farsipour and Zakerzadeh (2005), the estimator $c T_{m}+d$ is admissible, provided

(i) $0 \leqslant c<c_{1}, \quad d>0$,

(ii) $c=c_{1}, \quad d=0$,

where $c_{1}=m e^{-\Psi(m)}$ which is the value of $c$ that minimizes the risk function of $c T_{m}$. Therefore, the estimator $c_{1} T_{m}$ is admissible in the class of estimators of the form $c T_{m}$.

\section{Some Shrinkage Testimators}

In this section, we propose three shrinkage testimators and calculate their risks under the SLEL function. We construct our shrinkage testimators based on acceptance or rejection of $H_{0}: \theta=\theta_{0}$. The general form of the proposed shrinkage testimator is $k T_{m}+(1-k) \theta_{0}$, if $H_{0}: \theta=\theta_{0}$ is accepted or $c_{1} T_{m}$, otherwise. If $H_{0}: \theta=\theta_{0}$ is accepted at the level of $\alpha$, then we have

$$
\operatorname{Pr}\left(q_{1} \leqslant \frac{2 m T_{m}}{\theta_{0}} \leqslant q_{2}\right)=1-\alpha .
$$

Therefore, the proposed shrinkage testimators can be written as

$$
\hat{\theta}_{s t}^{(i)}= \begin{cases}k_{i} T_{m}+\left(1-k_{i}\right) \theta_{0} & t_{1} \leqslant T_{m} \leqslant t_{2} \\ c_{1} T_{m} & T_{m}<t_{1} \text { or } T_{m}>t_{2},\end{cases}
$$

where $t_{1}=q_{1} \theta_{0} / 2 m, t_{2}=q_{2} \theta_{0} / 2 m$ and $k_{i}, i=1,2,3$ are shrinkage factors corresponding to the shrinkage testimators $\hat{\theta}_{s t}^{(i)}, i=1,2,3$. In the sequel, we propose three shrinkage testimator for $\theta$.

\subsection{Shrinkage Testimator $\hat{\theta}_{s t}^{(1)}$}

The risk of the shrinkage estimator (5) under the SLEL function is

$$
R\left(\theta, \hat{\theta}_{S}\right)=E\left[\ln \frac{\hat{\theta}_{S}}{\theta}\right]^{2}=E\left[\ln \left(\frac{k T_{m}+(1-k) \theta_{0}}{\theta}\right)\right]^{2}
$$




$$
\begin{aligned}
& =E\left[\ln \left((1-k) \theta^{\star}+\frac{k W}{m}\right)\right]^{2} \\
& =\int_{0}^{\infty}\left[\ln \left((1-k) \theta^{\star}+\frac{k w}{m}\right)\right]^{2} g_{W}(w) d w
\end{aligned}
$$

where $\theta^{\star}=\theta_{0} / \theta$ and $W=m T_{m} / \theta$ with p.d.f. $g_{W}(w)=w^{m-1} e^{-w} / \Gamma(m)$. The value of $k_{1}=k_{\text {min }}$ which minimizes (12) can be obtained numerically and gives us the shrinkage testimator $\hat{\theta}_{s t}^{(1)}$.

\subsection{Shrinkage Testimator $\hat{\theta}_{s t}^{(2)}$}

If $H_{0}: \theta=\theta_{0}$ is accepted, then following Waikar et al. (1984), the inequality $q_{1} \leqslant 2 m T_{m} / \theta_{0} \leqslant q_{2}$ implies that

$$
0 \leqslant k_{2}=\frac{1}{q_{2}-q_{1}}\left(\frac{2 m T_{m}}{\theta_{0}}-q_{1}\right) \leqslant 1 .
$$

The value of $k_{2}$ can be used for constructing the shrinkage testimator $\hat{\theta}_{s t}^{(2)}$.

\subsection{Shrinkage Testimator $\hat{\theta}_{s t}^{(3)}$}

If $H_{0}: \theta=\theta_{0}$ is accepted, then following Prakash and Singh (2008), the inequality $q_{1} \leqslant 2 m T_{m} / \theta_{0} \leqslant q_{2}$ implies that $q_{1} \leqslant 2 m \leqslant q_{2}$ and then $q_{1} /(2 m) \leqslant 1$. For small values of shrinkage factor, we can take $q_{1} /(2 m) \approx 1$. Hence,

$$
\frac{2 m}{q_{2}-q_{1}}\left(\frac{2 m T_{m} / \theta_{0}}{2 m}-\frac{q_{1}}{2 m}\right) \approx \frac{2 m}{q_{2}-q_{1}}\left(\frac{T_{m}}{\theta_{0}}-1\right) .
$$

Therefore, the shrinkage factor $k_{3}$ for constructing the shrinkage testimator $\hat{\theta}_{s t}^{(3)}$ is given by

$$
k_{3}=\frac{2 m}{q_{2}-q_{1}}\left|\frac{T_{m}}{\theta_{0}}-1\right|
$$

where the absolute is for avoiding from negative values. 


\subsection{The Risks of Shrinkage Testimators $\hat{\theta}_{s t}^{(i)}$}

The risk of the shrinkage testimator $\hat{\theta}_{\text {st }}^{(i)}, i=1,2,3$ given in (11) under the LSEL function is

$$
\begin{aligned}
R\left(\theta, \hat{\theta}_{s t}^{(i)}\right) & =E\left[\ln ^{2}\left(\frac{\hat{\theta}_{s t}^{(i)}}{\theta}\right)\right] \\
& =E\left[\ln ^{2}\left(\frac{k_{i} T_{m}+\left(1-k_{i}\right) \theta_{0}}{\theta}\right) I\left(t_{1} \leqslant T_{m} \leqslant t_{2}\right)\right] \\
& +E\left[\ln ^{2}\left(\frac{c_{1} T_{m}}{\theta}\right) I\left(T_{m}<t_{1} \text { or } T_{m}>t_{2}\right)\right] \\
& =E\left[\ln ^{2}\left(\left(1-k_{i}\right) \theta^{\star}+\frac{k_{i} W}{m}\right) I\left(w_{1} \leqslant W \leqslant w_{2}\right)\right] \\
& +E\left[\ln ^{2}\left(\frac{c_{1} W}{m}\right)\right]-E\left[\ln ^{2}\left(\frac{c_{1} W}{m}\right) I\left(w_{1} \leqslant W \leqslant w_{2}\right)\right]
\end{aligned}
$$

where $W=m T_{m} / \theta, w_{1}=q_{1} \theta^{\star} / 2$ and $w_{2}=q_{2} \theta^{\star} / 2$. Using (9), we get $R\left(\theta, \hat{\theta}_{s t}^{(i)}\right)=\int_{w_{1}}^{w_{2}}\left\{\ln ^{2}\left(\left(1-k_{i}\right) \theta^{\star}+\frac{k_{i} w}{m}\right)-\ln ^{2}\left(\frac{c_{1} w}{m}\right)\right\} g(w) d w+\Psi^{\prime}(m)$,

which can be computed numerically using the statistical package $\mathrm{R}$ version 3.1.2.

Using a derivation similar to the above, we have

$$
E\left[\ln \left(\frac{\hat{\theta}_{s t}^{(i)}}{\theta}\right)\right]=\int_{w_{1}}^{w_{2}}\left\{\ln \left(\left(1-k_{i}\right) \theta^{\star}+\frac{k_{i} w}{m}\right)-\ln \left(\frac{c_{1} w}{m}\right)\right\} g(w) d w .
$$

For checking the condition of risk unbiasedness for $\hat{\theta}_{s t}^{(i)}$, we should prove that the expression given in (13) is zero, which is difficult to investigate theoretically, then we investigate it numerically. Figure 2, shows the plot of (13) for shrinkage testimator $\hat{\theta}_{s t}^{(1)}$ (solid line), $\hat{\theta}_{s t}^{(2)}$ (dashed line) and $\hat{\theta}_{s t}^{(3)}$ (dot) for selected values of $m=2,4$ and $\alpha=0.01,0.05,0.1$ with respect to $\theta^{\star}$ (more figures are provided, but not presented here). From Figure 2, we observe that the expression (13) may be negative, zero or positive, then we can state that the testimator $\hat{\theta}_{s t}^{(i)}$ may be negatively risk biased, risk unbiased or positively risk biased. 
$m=2, \alpha=0.01$

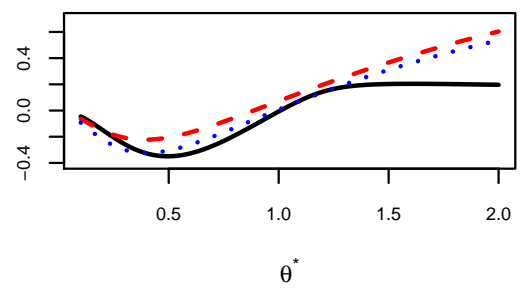

$m=2, \alpha=0.05$

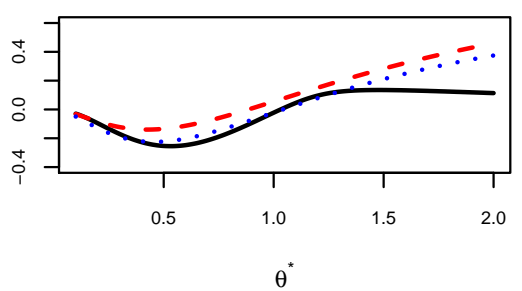

$m=2, \alpha=0.1$

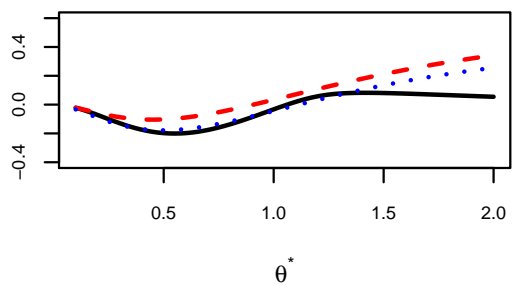

$m=4, \alpha=0.01$

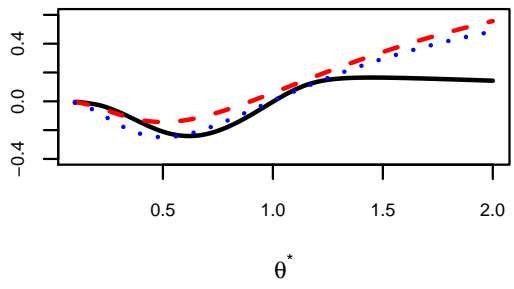

$\mathrm{m}=4, \alpha=0.05$

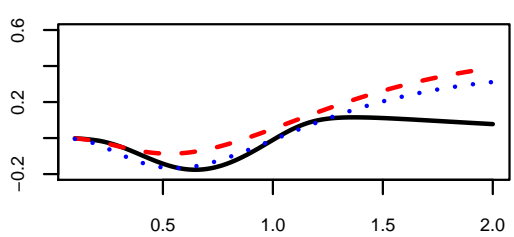

$m=4, \alpha=0.1$

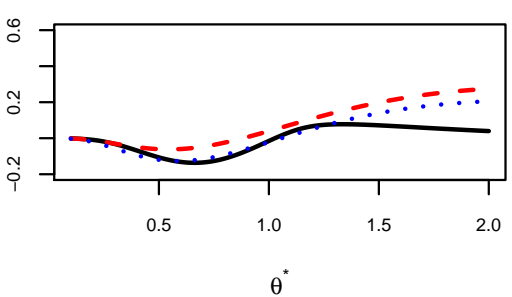

Figure 2. The plot of the expression (13) for shrinkage testimator $\hat{\theta}_{s t}^{(1)}$ (solid line), $\hat{\theta}_{s t}^{(2)}$ (dashed line) and $\hat{\theta}_{s t}^{(3)}(\mathrm{dot})$ for selected values of $m=2,4$ and $\alpha=0.01,0.05,0.1$ with respect to $\theta^{\star}$. 


\section{Comparison between Shrinkage Testimators and a Minimum Risk Unbiased Estimator}

In this section, we evaluate the performance of the proposed shrinkage testimators and the minimum risk unbiased estimator. For comparison, the relative efficiency (R.E.) of shrinkage testimator $\hat{\theta}_{s t}^{(i)}, i=1,2,3$ with respect to the minimum risk unbiased estimator $c_{1} T_{m}$ is calculated as

$$
R E\left(\hat{\theta}_{s t}^{(i)}, c_{1} T_{m}\right)=\frac{R\left(\theta, c_{1} T_{m}\right)}{R\left(\theta, \hat{\theta}_{s t}^{(i)}\right)}, \quad i=1,2,3 .
$$

Tables 1-3 give the relative efficiency (14) for the selected values of $m=$ $2(1) 5, \alpha=0.01,0.05,0.1$ and $\theta^{\star}=\theta_{0} / \theta=0.4(0.2) 1.8$. From these tables, we observe that no testimator performs uniformly better than the estimator $c_{1} T_{m}$. The testimator $\hat{\theta}_{s t}^{(1)}$ perform better than the estimator $c_{1} T_{m}$ in $0.8 \leqslant$ $\theta^{\star} \leqslant 1.8$. Also, the testimators $\hat{\theta}_{s t}^{(2)}$ and $\hat{\theta}_{s t}^{(3)}$ have good performance for $0.6 \leqslant \theta^{\star} \leqslant 1.4$ and $0.8 \leqslant \theta^{\star} \leqslant 1.4$, respectively.

For all testimators, the relative efficiency attains maximum at the point $\theta^{\star}=1$. For fixed $m$, as the value of $\alpha$ increases, the relative efficiency decreases for the testimators $\hat{\theta}_{s t}^{(1)}, \hat{\theta}_{s t}^{(2)}$ and $\hat{\theta}_{s t}^{(3)}$ in $0.6 \leqslant \theta^{\star} \leqslant 1.8,0.6 \leqslant \theta^{\star} \leqslant$ 1.4 and $0.6 \leqslant \theta^{\star} \leqslant 1.6$, respectively. The shrinkage testimator $\hat{\theta}_{s t}^{(1)}$ perform better than other shrinkage testimators when $0.8 \leqslant \theta^{\star} \leqslant 1.8$.

\section{A Real Example}

Consider a data set discussed by Dunsmore (1983). A rock crushing machine is kept working as long as the size of the crushed rock is larger than the rocks crushed before. Otherwise it is reset. The following data show the sizes of the crushed rocks up to the third reset of the machine:

$$
\begin{array}{lllllllllllll}
9.3 & 0.6 & 24.4 & 18.1 & 6.6 & 9.0 & 14.3 & 6.6 & 13 & 2.4 & 5.6 & 33.8 .
\end{array}
$$

The Kolmogorov-Smirnov (K-S) test was used for checking the validity of the exponential distribution based on the parameter $\theta=11.975$. It is observed that the $\mathrm{K}-\mathrm{S}$ distance is $\mathrm{K}-\mathrm{S}=0.2069$ with a corresponding $\mathrm{p}$-value $=0.6835$, which implies that the exponential distribution have a good fit to the above 
Table 1. Relative efficiency between $\hat{\theta}_{s t}^{(1)}$ and $c_{1} T_{m}$

\begin{tabular}{cccccccccc}
\hline \hline & & \multicolumn{7}{c}{$\theta^{\star}$} \\
\cline { 3 - 9 }$m=2$ & $\alpha$ & 0.4 & 0.6 & 0.8 & 1 & 1.2 & 1.4 & 1.6 & 1.8 \\
\hline \multirow{3}{*}{$m=.01$} & 0.9833 & 1.9467 & 5.0499 & 9.5744 & 6.3681 & 4.0207 & 3.0760 & 2.5961 \\
& 0.05 & 0.9026 & 1.4103 & 2.4175 & 3.1995 & 2.7920 & 2.2128 & 1.8635 & 1.6526 \\
& 0.1 & 0.8814 & 1.2173 & 1.7593 & 2.1167 & 1.9803 & 1.7143 & 1.5224 & 1.3966 \\
& 0.01 & 0.8309 & 1.4632 & 4.1203 & 10.2077 & 5.5089 & 3.1907 & 2.3946 & 2.0062 \\
$m=3$ & 0.05 & 0.8107 & 1.1706 & 2.2094 & 3.3140 & 2.6379 & 1.9333 & 1.5840 & 1.3933 \\
& 0.1 & 0.8189 & 1.0659 & 1.6646 & 2.1656 & 1.9146 & 1.5602 & 1.3526 & 1.2336 \\
& 0.01 & 0.7866 & 1.2230 & 3.4745 & 10.5717 & 4.8151 & 2.6822 & 2.006 & 1.6817 \\
$m=4$ & 0.05 & 0.7925 & 1.0353 & 2.0250 & 3.3769 & 2.4752 & 1.7296 & 1.4053 & 1.2398 \\
& 0.1 & 0.8123 & 0.9747 & 1.5738 & 2.1919 & 1.8387 & 1.4400 & 1.2390 & 1.1349 \\
& 0.01 & 0.7806 & 1.0847 & 3.0158 & 10.8050 & 4.2798 & 2.3430 & 1.7569 & 1.4775 \\
$m=5$ & 0.05 & 0.8017 & 0.9532 & 1.8710 & 3.4163 & 2.3273 & 1.5795 & 1.2840 & 1.1414 \\
& 0.1 & 0.8268 & 0.9179 & 1.4933 & 2.2083 & 1.7657 & 1.3474 & 1.1602 & 1.0719 \\
& .01 & 0.7899 & 0.9978 & 2.6773 & 10.9664 & 3.8625 & 2.1011 & 1.5830 & 1.3377 \\
$m=6$ & .05 & 0.8209 & 0.9013 & 1.7433 & 3.4431 & 2.1974 & 1.4655 & 1.1974 & 1.0450 \\
& 0.1 & 0.8484 & 0.8821 & 1.4232 & 2.2193 & 1.6988 & 1.2750 & 1.1037 & 1.0303 \\
\hline
\end{tabular}

Table 2. Relative efficiency between $\hat{\theta}_{s t}^{(2)}$ and $c_{1} T_{m}$

\begin{tabular}{cccccccccc}
\hline \hline & & \multicolumn{7}{c}{$\theta^{\star}$} \\
\cline { 3 - 9 }$m=2$ & 0.4 & 0.6 & 0.8 & 1 & 1.2 & 1.4 & 1.6 & 1.8 \\
\hline \multirow{3}{*}{$m=3$} & 0.01 & 1.1153 & 2.0508 & 3.7914 & 5.1386 & 4.3388 & 2.9813 & 2.0581 & 1.5007 \\
& 0.05 & 1.0436 & 1.5365 & 2.1740 & 2.5010 & 2.2775 & 1.8346 & 1.4428 & 1.1547 \\
& 0.1 & 1.0089 & 1.3239 & 1.6746 & 1.8356 & 1.7266 & 1.4858 & 1.2449 & 1.0494 \\
& 0.01 & 0.9059 & 1.5886 & 3.1420 & 4.6664 & 3.7103 & 2.2956 & 1.4779 & 1.0343 \\
& 0.05 & 0.9153 & 1.3220 & 2.0059 & 2.4242 & 2.0983 & 1.5508 & 1.1407 & 0.8760 \\
& 0.1 & 0.9240 & 1.2009 & 1.6030 & 1.8073 & 1.6307 & 1.3102 & 1.0381 & 0.8454 \\
& 0.01 & 0.8157 & 1.3430 & 2.7396 & 4.3580 & 3.2849 & 1.8770 & 1.1551 & 0.7903 \\
$m=4$ & 0.05 & 0.8598 & 1.1885 & 1.8774 & 2.3649 & 1.9540 & 1.3487 & 0.9503 & 0.7159 \\
& 0.1 & 0.8875 & 1.1175 & 1.5432 & 1.7837 & 1.5489 & 1.1777 & 0.9005 & 0.7234 \\
& 0.01 & 0.7734 & 1.1911 & 2.4628 & 4.1423 & 2.9709 & 1.5918 & 0.9492 & 0.6412 \\
$m=5$ & 0.05 & 0.8370 & 1.0984 & 1.7757 & 2.3191 & 1.8352 & 1.1979 & 0.8206 & 0.6142 \\
& 0.1 & 0.8744 & 1.0581 & 1.4925 & 1.7847 & 1.4789 & 1.0748 & 0.8039 & 0.6450 \\
& 0.01 & 0.7552 & 1.0882 & 2.2583 & 3.9825 & 2.7251 & 1.3837 & 0.8065 & 0.5414 \\
$m=6$ & 0.05 & 0.8313 & 1.0342 & 1.6923 & 2.2829 & 1.7347 & 1.0810 & 0.7273 & 0.5454 \\
& 0.1 & 0.8738 & 1.0144 & 1.4487 & 1.7493 & 1.4180 & 0.9929 & 0.7331 & 0.5922 \\
\hline
\end{tabular}


Table 3. Relative efficiency between $\hat{\theta}_{s t}^{(3)}$ and $c_{1} T_{m}$

\begin{tabular}{cccccccccc}
\hline \hline & & \multicolumn{7}{c}{$\theta^{\star}$} \\
\cline { 3 - 9 }$m=2$ & $\alpha$ & 0.4 & 0.6 & 0.8 & 1 & 1.2 & 1.4 & 1.6 & 1.8 \\
\hline \multirow{3}{*}{$m=3$} & 0.01 & 0.9855 & 1.9553 & 4.1208 & 6.4123 & 5.4912 & 3.6261 & 2.4394 & 1.7592 \\
& 0.1 & 0.9221 & 1.4345 & 2.2102 & 3.7509 & 2.5994 & 2.1127 & 1.6666 & 1.3397 \\
& 0.8993 & 1.2281 & 1.6566 & 1.9345 & 1.8964 & 1.6674 & 1.4150 & 1.2052 \\
$m=3$ & 0.05 & 0.7788 & 1.2001 & 2.0505 & 2.7665 & 2.4691 & 1.8171 & 1.3370 & 1.0344 \\
& 0.1 & 0.8012 & 1.0861 & 1.5822 & 1.9469 & 1.8379 & 1.5008 & 1.2017 & 0.9899 \\
& 0.01 & 0.6562 & 1.2086 & 3.0762 & 6.2553 & 4.3426 & 2.2675 & 1.3683 & 0.9416 \\
$m=4$ & 0.05 & 0.7121 & 1.0495 & 1.9152 & 2.7706 & 2.3367 & 1.5912 & 1.1238 & 0.8572 \\
& 0.1 & 0.7568 & 0.9879 & 1.5147 & 1.9522 & 1.7739 & 1.3637 & 1.0537 & 0.8582 \\
& 0.01 & 0.6008 & 1.0377 & 2.7568 & 6.2048 & 3.9189 & 1.9092 & 1.1279 & 0.7733 \\
$m=5$ & 0.05 & 0.6812 & 0.9453 & 1.7998 & 2.7715 & 2.2138 & 1.4183 & 0.9780 & 0.7447 \\
& 0.1 & 0.7397 & 0.9169 & 1.4540 & 1.9550 & 1.7116 & 1.2528 & 0.9481 & 0.7730 \\
& 0.01 & 0.5713 & 0.9189 & 2.5059 & 6.1664 & 3.5701 & 1.6512 & 0.9639 & 0.6618 \\
$m=6$ & 0.05 & 0.6703 & 0.8697 & 1.7002 & 2.7712 & 2.1023 & 1.2831 & 0.8733 & 0.6689 \\
& 0.1 & 0.7382 & 0.8641 & 1.3994 & 1.9565 & 1.6529 & 1.1626 & 0.8704 & 0.7157 \\
\hline
\end{tabular}

data. The observed upper record values $\mathbf{r}=\left(r_{1}, r_{2}, r_{3}\right)$ are obtained to be

$$
\mathbf{r}=(9.3,24.4,33.8)
$$

Then, the MLE is $T_{3}=\frac{r_{3}}{3}=11.27$. Also, we have $c_{1}=3 e^{-\Psi(3)}=1.19$, which indicates that a minimum risk unbiased estimator $c_{1} T_{3}$ is 13.44. We consider the estimation of $\theta$ when the guess value is $\theta_{0}=11$. Using the ML estimate of $\theta$, the estimate of $\theta^{\star}$ is $\hat{\theta}^{\star}=\frac{\theta_{0}}{T_{3}}=0.98$ and therefore the value of shrinkage factor $k_{1}$ founded by minimizing the risk of shrinkage estimator $\hat{\theta}_{S}$ given in (5) is 0.001 . The test statistic for testing the null hypothesis $H_{0}: \theta=11$ is $\chi^{2}=6.15$. If we consider $\alpha=0.05$, then the left quantiles of a chi-square distribution with 6 degree of freedom are $q_{1}=1.24$ and $q_{2}=14.45$. This implies that the null hypothesis is accepted. Then the values of shrinkage factors $k_{2}$ and $k_{3}$ are as follows:

$$
\begin{aligned}
& k_{2}=\frac{1}{14.45-1.24}\left(\frac{2(3) 11.27}{11}-1.24\right)=0.37 \\
& k_{3}=\frac{2(3)}{14.45-1.24}\left|\frac{11.27}{11}-1\right|=0.009 .
\end{aligned}
$$


Table 4. Risks and relative efficiency of estimators

\begin{tabular}{ccccc}
\hline \hline estimator & $c_{1} T_{3}$ & $\hat{\theta}_{s t}^{(1)}$ & $\hat{\theta}_{s t}^{(2)}$ & $\hat{\theta}_{s t}^{(3)}$ \\
\hline Risk & 0.39493 & 0.12019 & 0.15141 & 0.12021 \\
R.E. & & 3.28555 & 2.60817 & 3.28494 \\
\hline
\end{tabular}

Using the values of shrinkage factors $k_{i}, i=1,2,3$, we obtain the risks of shrinkage testimators $\hat{\theta}_{s t}^{(i)}$ given in (11) and relative efficiency of them with respect to $c_{1} T_{3}$ which are summarized in Table 4 .

From Table 4, we observe that all of the shrinkage testimators are better than the estimator $c_{1} T_{3}$. Also, the shrinkage testimator $\hat{\theta}_{s t}^{(1)}$ is more efficient than other shrinkage testimators, however it is comparable with the testimator $\hat{\theta}_{s t}^{(3)}$.

\section{Concluding Remarks}

The problem of shrinkage testimation under the squared log error loss function on the basis of observed exponential records is considered. Some shrinkage testimators are provided and their risks are computed. Comparisons are made between these testimators and a minimum risk unbiased estimator within the class of estimators of the form $c T_{m}$. The results show that the shrinkage testimators are more efficient when the experimenter has a priori that the guess value $\theta_{0}$ is in the vicinity of $\theta$. Also, the shrinkage testimator $\hat{\theta}_{s t}^{(1)}$ corresponding to the shrinkage factor $k_{1}$, which founded by minimizing the risk of the shrinkage estimator $\hat{\theta}_{S}$, performs better than other shrinkage testimators for more values of $\theta^{\star}=\frac{\theta_{0}}{\theta}$. Finally, we presented a real data set to illustrate the results. Note that the shrinkage factor $k_{1}$ which constructs the testimator $\hat{\theta}_{s t}^{(1)}$, depends upon the unknown parameter $\theta$, hence an estimate $\hat{k}_{1}$ of $k_{1}$ can be obtained by replacing the parameter $\theta$ to ML estimator.

\section{Acknowledgement}

The authors are grateful to the Editor and two reviewers for making helpful comments and suggestions on an earlier version of this paper. 


\section{References}

Arnold, B.C., Balakrishnan, N. and Nagaraja, H.N. (1998). Records. New York, John Wiley.

Brown, L.D. (1990). Comment on Maatta and Casella's Paper. Statistical Science, 5, 103-106.

Dunsmore, I.R. (1983). The Future Occurrence of Records. Annals of the Institute of Statistical Mathematics 35, 267-277.

Kiapour, A. and Nematollahi, N. (2011). Robust Bayesian Prediction and Estimation under a Squared Log Error Loss function. Statistics and Probability Letters, 81, 1717-1724.

Lehmann, E.L. (1951). A General Concept of Unbiasedness. Annals of Mathematical Statistica, 22, 587-592.

Pal, N. and Ling, C. (1996). Estimation of a Normal Scale Parameter under a Balanced Loss Function. Journal of the Indian Statistical Association, 34, 21-38.

Prakash, G., and Singh, D.C. (2008). Shrinkage Estimation in Exponential Type-II Censored Data under LINEX Loss. Journal of the Korean Statistical Society, 37, 53-61.

Sanjari Farsipour, N. and Zakerzadeh, H. (2005). Estimation of a Gamma Scale Parameter under Asymmetric Squared Log Error Loss, Communications in Statistics-Theory and Methods, 34, 1127-1135.

Thompson, J.R. (1968). Some Shrunken Techniques for Estimating the Mean. Journal of the American Statistical Association,. 63, 113-122.

Waikar, V.B., Schuurmann, F. J. and Raghunathan, T.E. (1984). On a Two Stage Shrunken Testimator of the Mean of a Normal Distribution. Communications in Statistics-Theory and Methods, 13, 1901-1913.

\section{Naghizadeh Qomi}

Department of Statistics,

University of Mazandaran,

Babolsar, Iran.

email:m.naghizadeh@umz.ac.ir

\section{Barmoodeh}

Department of Statistics,

University of Mazandaran,

Babolsar, Iran.

email: k.mehraneh@chmail.ir 
